\title{
Robotic-assisted magnetic resonance imaging ultrasound fusion results in higher significant cancer detection compared to cognitive prostate targeting in biopsy naive men
}

\author{
Manish I. Patel ${ }^{1,2}$, Samir Muter ${ }^{2}$, Philip Vladica ${ }^{3}$, David Gillatt ${ }^{2}$ \\ ${ }^{1}$ Discipline of Surgery, University of Sydney, Sydney, Australia; ${ }^{2}$ Department of Surgery, Macquarie University Hospital, Sydney, Australia; \\ ${ }^{3}$ Department of Radiology, Westmead Hospital, Sydney, Australia \\ Contributions: (I) Conception and design: All authors; (II) Administrative support: None; (III) Provision of study material or patients: MI Patel; (IV) \\ Collection and assembly of data: S Muter, MI Patel; (V) Data analysis and interpretation: MI Patel, S Muter; (VI) Manuscript writing: All authors; (VII) \\ Final approval of manuscript: All authors. \\ Correspondence to: Prof. Manish I. Patel, MBBS, MMed, PhD, FRACS. University of Sydney, Suite 17 Westmead Private Hospital, Westmead, NSW \\ 2145, Australia. Email: mpatel@med.usyd.edu.au.
}

\begin{abstract}
Background: To determine differences in cancer detection rates (CDRs) of regions of interest (ROI) on magnetic resonance imaging (MRI) with robotic-assisted (RA) targeted biopsies (RA-TB) compared to cognitive targeted biopsies (C-TB).

Methods: In a two-centre, retrospective outcome study, a total of 92 consecutive men who had a pre-biopsy MRI, were biopsy naïve and had a transperineal (TP) prostate biopsy between 9/2015 and 7/2017 were included. The primary analysis consists of 39 men who had C-TB and 53 men who had RA-TB. Outcomes from targeted biopsies were reported as CDR for clinically significant prostate cancer (csPC) and for any cancer.

Results: Targeted csCDR was higher in RA-TB vs. C-TB (32.1\% vs. 10.3\%, P=0.014). Targeted CDR of any cancer with Prostate Imaging Reporting and Data System (PI-RADS) 3-5 ROIs was also significantly higher in RA-TB compared to C-TB $(47.2 \%$ vs. $12.8 \%, \mathrm{P}=0.001)$. On multivariable analysis significant factors which affected the CDR for csPC was prostate volume only [odds ratio (OR) 1.04, 95\% confidence interval (CI): 1.01-1.07]. For any cancer, the CDR was related to prostate volume (OR 1.03, 95\% CI: $1.01-$ 1.06) and RA-TB (OR 5.97, 95\% CI: 1.69-21.07). RA biopsy results in less acute urinary retention $(1.9 \%$ vs. $12.8 \%, \mathrm{P}=0.03)$, less prolonged haematuria $(7.5 \%$ vs. $38.5 \%, \mathrm{P}<0.01)$ and shortened biopsy times $(24$ vs. $32 \mathrm{~min}, \mathrm{P}<0.001)$.
\end{abstract}

Conclusions: RA targeted biopsy results in higher CDR for clinically significant cancers and any cancer. It also suffers lower complications compared to cognitive directed TP biopsy. The ease of use and standardisation of the robotic procedure may reduce the learning curve and increase biopsy accuracy.

Keywords: Prostate biopsy; robotic-assisted (RA); targeted biopsy; fusion biopsy; cognitive biopsy

Submitted Sep 21, 2019. Accepted for publication Jan 06, 2020.

doi: $10.21037 /$ tau.2020.01.33

View this article at: http://dx.doi.org/10.21037/tau.2020.01.33

\section{Introduction}

Diagnosis of prostate cancer (PC), following the detection of an elevated prostate-specific antigen (PSA) test has evolved over the last 5 years. Imaging with multiparametric $(\mathrm{mp})$ magnetic resonance imaging (MRI) prior to a biopsy has resulted in the potential to increase the cancer detection rate (CDR) of clinically significant PC (csPC) while decreasing the insignificant $\mathrm{CDR}$ and number of unnecessary biopsies $(1,2)$.

There is still controversy over how to best perform 
prostate biopsies in order to maximise csPC detection whilst minimising the detection of insignificant cancers. A systematic review reported by Wegelin et al. which included 43 studies, concluded that in-bore biopsy was superior to cognitive targeted biopsies (C-TB) for the detection of all $\mathrm{PC}$ however there were no differences in the detection of csPC between C-TB, MRI ultrasound fusion biopsy (MFB) and in-bore MRI targeted biopsies (3). Another study from New York University compared C-TB to $\mathrm{MFB}$ and reported that $\mathrm{MFB}$ detected higher csPC rates compared to $\mathrm{C}-\mathrm{TB}$ alone however this was not statistically significant (4).

There is also controversy over the best approach to biopsy which can also be performed transrectally (TR) or transperineally (TP), where TR has the advantage of being simple and office based, it does experience the disadvantage of potential uro-sepsis which appears to be increasing in rate (5). TP biopsy on the other hand, has the disadvantage of requiring a general anaesthetic in many instances, although there are now many reports that this can be performed under local anaesthesia (6).

The primary aim of this study is to determine the csCDR of TP targeted biopsies using robotic-assisted (RA) MFB compared to C-TB using a grid template as this has not previously been reported. Secondary aims are to determine differences in complications between the two methods.

\section{Methods}

Consecutive patients between September 2015 and July 2017 who had a suspicion of PC based on their PSA levels or digital rectal exam (DRE) underwent a 3.0T mpMRI with body coil. The method has been previously described, with the modification of omitting spectroscopy (7). T2weighted, diffusion weighted and dynamic contrast enhanced images were performed. Patients with previous prostate biopsies or contra-indication to mpMRI were excluded.

All mpMRIs were performed by one experienced radiologist (P Vladica) and reported using the Prostate Imaging Reporting and Data System (PI-RADS), version 2.0 (8). Regions of interest (ROI) (PI-RADS 3-5) were demonstrated on a prostate diagram and dimensions measured in $\mathrm{mm}$. Representative images of all suspicious lesions were also provided to the urologist. Only men with ROIs (PI-RADS 3-5) and underwent a TP targeted biopsy (RA-MFB or C-TB) and were included in this study. These men all received a general anaesthetic to ensure lack of movement and antibiotic prophylaxis.

All biopsies were performed by one surgeon (MI Patel) who had over 17 years of experience with TP prostate biopsies and 2 years with C-TB.

\section{RA-MRI-US fusion prostate biopsy}

A detailed method of RA-MFB has been described by Mischinger et al. (9). Briefly the prostate outline and ROIs were contoured semi-automatically by the urologist using image fusion software (UrofusionTM, Biobot Surgical, Singapore, Singapore). The patient was placed in lithotomy and the perineum prepped. The ultrasound probe (BK 8848; BK Medical, Peabody, MA, USA) was inserted TR and prostate images acquired at $1 \mathrm{~mm}$ slice thickness. The prostate outlines were contoured semi-automatically and the previously contoured MRI images imported and elastic fusion performed which compensates for organ deformity (UroBiopsyTM; Biobot Surgical). Target ROIs had between 2-6 biopsy directed cores depending on lesion size. The TB were automatically programmed based on lesion/prostate shape and size but the location was able to be modified. Needle direction, penetration depth and biopsy position were determined by the robotic system and taken with an 18-G biopsy needle. Only one puncture site was made per prostate lobe.

\section{$C-T B$}

The cognitive directed (non-RA) TB were performed using a brachytherapy template grid with 5 -mm spacing and a BK Medical (BK Ultrasound, Peabody, MA, USA) FlexFocusTM ultrasound with 8848 transrectal probe mounted on a Civco (Kalona, IA, USA) MicroTouchTM stepper. Targeted Biopsies were performed by 2-6 cores depending on the lesion size. Following this, all underwent sector biopsies (SB) by the Ginsburg protocol $(10,11)$.

The mean duration of each procedure was measured from the time of rectal probe insertion to the time of probe removal. For the RA biopsy, time was required for the contouring of the MRI images which were completed in the time during patient change over and was not measured but typically took $10 \mathrm{~min}$.

Post-operative complications were measured by the Clavien-Dindo classification $(12,13)$ and also reported in terms of sepsis, haematuria and acute urinary retention. 
Table 1 Clinical characteristics of patients included in the study

\begin{tabular}{|c|c|c|c|}
\hline Characteristic & Cognitive-targeted & Robotic-assisted targeted & Total \\
\hline Age (years), median [IQR] & 62 [56-68] & 65 [61-69] & 63 [58-68] \\
\hline \multicolumn{4}{|l|}{ PSA (ng/mL), n (\%) } \\
\hline$<4$ & $9(23.1)$ & $11(20.8)$ & $20(21.7)$ \\
\hline$>10$ & $8(20.5)$ & $2(3.8)$ & $10(10.9)$ \\
\hline \multicolumn{4}{|l|}{ DRE, n (\%) } \\
\hline Negative & $23(59.0)$ & $34(64.2)$ & $57(62.0)$ \\
\hline Positive & $16(41.0)$ & $19(35.8)$ & $35(38.0)$ \\
\hline PI-RADS 3 & $14(38.5)$ & $14(26.4)$ & $28(30.4)$ \\
\hline PI-RADS 4 & $13(33.3)$ & $28(52.8)$ & $41(44.6)$ \\
\hline PI-RADS 5 & $11(28.2)$ & $11(20.8)$ & $22(23.9)$ \\
\hline Major lesion size $(\mathrm{mm})$, median (IQR) & $10.5(7.0-16.0)$ & $12.9(6.0-17.5)$ & $12.0(7.0-17.5)$ \\
\hline Target cores $\mathrm{n}$, median [IQR] & $3[2-3]$ & $4[3-5]$ & $3[2.25-4]$ \\
\hline Time (min), median [IQR] & 32 [31-36] & 24 [21-28] & 28 [27-32] \\
\hline
\end{tabular}

IQR, interquartile range; PSA, prostate-specific antigen; DRE, digital rectal exam; PI-RADS, Prostate Imaging Reporting and Data System.

\section{Histopathology}

All pathology specimens were read by a single experienced uro-pathologist and grade apportioned using the international society of uro-pathologist (ISUP) 2014 classification system (13). Clinically significant cancer was defined as ISUP grade group 2 or higher.

\section{Statistics}

All data was collected prospectively. Patients with multiple ROIs, the ROI with the highest PI-RADs score was considered the index lesion and used for analysis. Dichotomous variables were compared with the twosided Fisher's exact test and continuous variables with the Mann-Whitney U-test. A $\mathrm{P}<0.05$ was considered as the level of significant difference. Multivariable analysis was performed by logistic regression analysis. Statistical analysis was performed with SPSS ver 24 (IBM Corp., Armonk, NY, USA).

The Macquarie University Human Ethics Committee approved this study (5201700919).

\section{Results}

The clinical characteristics of patients included in this study are given in Table 1. A total of 92 patients were included of which 39 received C-TB and 53 received RA-TB.

Of these patients $20.5 \%$ having C-TB had a PSA higher than $10 \mathrm{ng} / \mathrm{mL}$ compared to $3.8 \%$ with RA-TB. In addition, $28.2 \%$ of lesions were PI-RADS 5 with C-TB compared to $20.8 \%$ with RA-TB. The median maximum diameters of ROIs were similar (10.5 vs. $12.9 \mathrm{~mm})$. A similar number of targeted cores were also taken in both the C-TB and RATB cohorts (3 vs. 4).

\section{PC detection: targeted biopsies}

Figure $1 A$ describes the csCDR (defined as ISUP grade 2 or above) from targeted ROIs (PI-RADS 3-5). Clinically significant cancer was significantly higher for RA-TB 17/53 (32.1\%) compared to C-TB 4/39 (10.3\%) ( $\mathrm{P}=0.014)$ (Table 2). Maximum cancer core length per patient was $6.21 \mathrm{~mm}$ for RA-TB compared to $6.46 \mathrm{~mm}$ for C-TB $(\mathrm{P}=0.13)$.

For all cancers (ISUP 1-5) RA-TB was also higher 25/53 

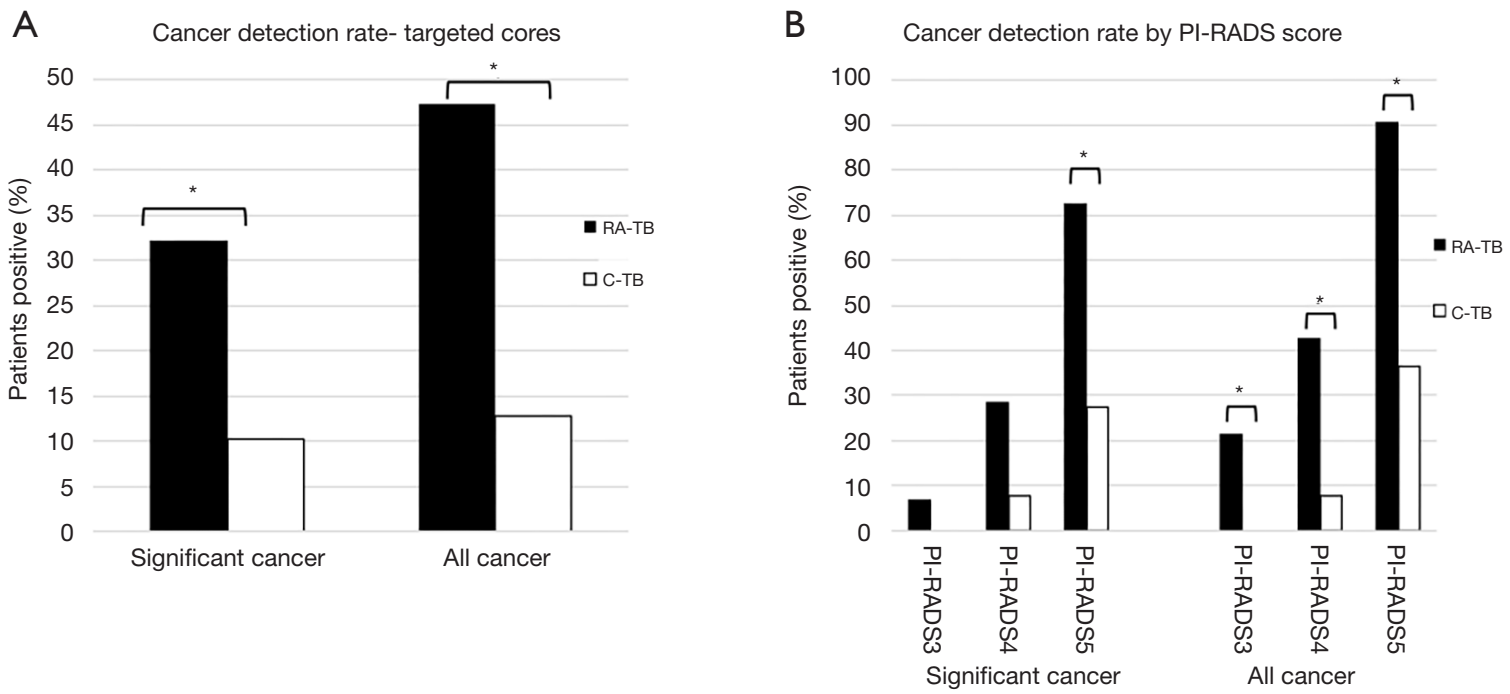

Figure 1 Cancer detections rates from (A) targeted cores stratified by significant and all cancers and (B) from targeted cores stratified by PI-RADS score and by significant and all cancers. *, $\mathrm{P}<0.05$. RA-TB, robotic-assisted targeted biopsy; C-TB, cognitive targeted biopsies; PI-RADS, Prostate Imaging Reporting and Data System.

Table 2 Cancer detection rates for robotic-assisted compared to cognitive targeted biopsies

\begin{tabular}{|c|c|c|c|}
\hline Variable & RA-TB, n (\%) (n=53) & C-TB, n (\%) (n=39) & $P$ value \\
\hline Clinically significant cancer & $17(32.1)$ & $4(10.3)$ & 0.014 \\
\hline Maximum cancer core length (mm) & 6.21 & 6.46 & 0.13 \\
\hline All cancers & $25(47.2)$ & $5(12.8)$ & 0.001 \\
\hline Insignificant cancer & $8(15.1)$ & $1(2.6)$ & 0.05 \\
\hline Maximum cancer core length (mm) & 4.38 & 5.10 & 0.53 \\
\hline \multicolumn{4}{|l|}{ Systematic biopsy } \\
\hline Clinically significant cancer & $4(7.5)$ & $10(25.6)$ & 0.02 \\
\hline Insignificant cancer & $3(5.7)$ & $2(5.1)$ & 0.91 \\
\hline Maximum cancer core length (mm) & 2.17 & 2.43 & 0.72 \\
\hline \multicolumn{4}{|l|}{ Targeted and systematic } \\
\hline Clinically significant cancer & $21(39.6)$ & $14(35.9)$ & 0.72 \\
\hline All cancers & $32(60.4)$ & $17(43.6)$ & 0.11 \\
\hline
\end{tabular}

RA-TB, robotic-assisted targeted biopsies; C-TB, cognitive targeted biopsies. 
Table 3 Multivariable analysis of factors associated with a positive biopsy

\begin{tabular}{lcc}
\hline \multirow{2}{*}{ Variable } & \multicolumn{2}{c}{ Odds ratio $(95 \% \mathrm{Cl})$} \\
\cline { 2 - 3 } & Significant cancer & All cancers \\
\hline Age (years) & $0.94(0.86-1.02)$ & $0.99(0.92-1.08)$ \\
Prostate volume $(\mathrm{mL})$ & $1.04(1.01-1.07)^{\star}$ & $1.03(1.01-1.06)^{\star}$ \\
PSA $(\mathrm{ng} / \mathrm{mL})$ & $0.97(0.82-1.15)$ & $0.99(0.85-1.15)$ \\
RA-TB (reference: C-TB) & $3.40(0.79-14.62)$ & $5.97(1.69-21.07)^{\star}$ \\
Targeted cores $(\mathrm{n})$ & $0.74(0.52-1.05)$ & $0.76(0.55-1.06)$ \\
\hline
\end{tabular}

*, $\mathrm{P}<0.05$. Cl, confidence interval; PSA, prostate-specific antigen; RA-TB, robotic-assisted targeted biopsies; C-TB, cognitive targeted biopsies.

(47.2\%) compared to C-TB 5/39 (12.8\%) ( $\mathrm{P}=0.001)$ (Table 2). Maximum cancer core length per patient was $5.92 \mathrm{~mm}$ for RA-TB compared to $5.84 \mathrm{~mm}$ for $\mathrm{C}-\mathrm{TB}(\mathrm{P}=0.34)$.

Insignificant cancer, defined as ISUP grade 1 was $8 / 53$ (15.1\%) for RA-TB and $1 / 39$ (2.6\%) for C-TB $(\mathrm{P}=0.05)$ (Table 2). Maximum cancer core length per patient was $4.38 \mathrm{~mm}$ for RA-TB compared to $5.10 \mathrm{~mm}$ for C-TB $(\mathrm{P}=0.53)$.

\section{PI-RADS score and cancer detection}

Overall, as the PI-RADS score of ROIs rose from 3 to 5, clinically significant and total CDRs increased (Figure 1B). For significant cancers PI-RADS 5 was associated with a $73 \% \mathrm{CDR}$ compared to $27 \%$ with $\mathrm{C}-\mathrm{TB}(\mathrm{P}=0.02)$. For all cancers there was also a significant difference in $\mathrm{CDR}$ with $91 \%$ for RA-TB and 36\% for C-TB for PI-RADS 5 lesions $(\mathrm{P}<0.01)$.

\section{Multivariable analysis for detection of cancer on targeted biopsy}

Table 3 outlines the impact of various factors on the likelihood of the detection of any cancer. In this model, age was not a significant factor, however for the detection significant cancer, prostate volume was a significant factor (OR 1.04, 95\% CI: 1.01-1.07). For the detection of all cancers, Prostate volume (OR 1.03, 95\% CI: 1.01-1.06) and RA-TB (OR 5.97, 95\% CI: 1.69-21.07) were significantly associated.

\section{Complications following TP prostate biopsies}

There were significantly more episodes of acute retention following C-TB, 5/39 (12.8\%) compared to 1/53 (1.9\%) for RA biopsy $(\mathrm{P}=0.03)$. Haematuria lasting longer than 3 days was also higher in the template directed group 15/39 (38.5\%) compared to $4 / 53$ (7.5\%) for the robot assisted group $(\mathrm{P}<0.01)$. Patient complaints of perineal bruising was similar $(8.8 \%$ vs. $8.0 \%)$. There were no episodes of urinary tract infection (UTI) or sepsis in either group.

\section{Time for procedures}

It was anticipated that using the robotic system would lead to substantially longer procedure times, which were calculated from the time of probe insertion to probe removal. Table 1 outlines the times taken for the procedures. These times do not include the time taken to contour the prostate and the ROI outlines for RA biopsies, which was typically completed during change over and anaesthesia induction and took approximately $10 \mathrm{~min}$.

The time taken for C-TB was significantly longer than RA-TB (32 vs. 24 min, $\mathrm{P}<0.001$ ).

\section{Discussion}

Recent studies have demonstrated that performing a MRI prior to targeted prostate biopsy results in higher significant CDRs compared to standard transrectal systematic biopsies $(1,2,14)$. The PRECISION study findings show that targeted biopsies compared to systematic TRUS biopsies result in higher csCDR and lower insignificant CDRs (2). How to perform targeted biopsy, following an MRI which shows suspicious ROIs, is an increasing controversy. Targeted biopsies may be performed TR or TP and by cognitive registration, MRI-US fusion or inbore. Numerous studies have been published to identify 
the relative merits of each approach, however there is no clear approach which stands out. A systematic review by Wegelin et al. identified 11 studies using in-bore TB, 17 MRI fusion targeted biopsy (MF-TB) and 11 using C-TB and 4 with a combination (3). In that study, in-bore TB was not significantly different to MF-TB and MF-TB was no different to C-TB. A study from Memorial Sloan-Kettering Cancer Center, also found no difference between MFTB and C-TB. However, a very well-conducted study by Wysock et al. (4) compared MRI-US fusion targeting, using the Artemis system, to C-TB and reported total CDR of the targets to be $32 \%$ compared to $27 \%$ for MF-TB vs. C-TB and $20 \%$ vs. $15 \%$ for csPC respectively. These were not statistically significant, but do suggest with larger numbers there may have been a difference.

Our study is the first examine the differences in CDR of the targeted ROI between RA-TB and C-TB. The results have shown a clear difference in CDR of PI-RADS 3-5 ROIs, with $32.1 \%$ vs. $10.3 \%$ ( $\mathrm{P}=0.014)$ for csPC and $47 \%$ vs. $13 \%(\mathrm{P}=0.001)$ for all $\mathrm{PC}$ and with $\mathrm{RA}-\mathrm{TB}$ compared to $\mathrm{C}-\mathrm{TB}$ respectively.

While the CDR with C-TB in our study was low and possibly due to inexperience, these biopsies were performed by an experienced urologist who has been performing $\mathrm{TP}$ prostate biopsies for 17 years and C-TB for 2 years prior to the start of this study. In the study by Wysock et al. (4), C-TB were performed by a urologist with one of the world's largest experiences in the technique and demonstrated a CDR of $34 \%$. Globally very few urologists would have this level of experience, and hence in the real world our CDR with C-TB is probably appropriate. What is not clear is whether the addition of RA technology has improved the CDR of MFTB or whether MF-TB alone is superior to C-TB.

Multivariable analysis has shown that RA biopsy was an independent significant factor in determining a positive cancer diagnosis from targeted cores [OR 5.97, 95\% CI: 1.69-21.07] in addition to prostate volume.

As the PI-RADS score of the MRI ROIs increased, so did the CDR. For csPC it ranged from $7-73 \%$ and $0-27 \%$ for RA-TB and C-TB respectively.

The csPC and all CDR of systematic biopsies was superior in the C-TB compared to RA-TB group (Table 2). The majority of these positive biopsies were in the sector of the ROI, suggesting that systematic biopsies can compensate for the lower accuracy obtained from C-TB. A recent study by Miah et al. examined the CDR in 86 men biopsied using the same robotic biopsy platform (15). In total, $51 \%$ were diagnosed with clinically significant cancer- slightly higher than $40 \%$ in our series. They also reported that systematic biopsies diagnosed a further $10.5 \%$ csPC over targeted biopsies alone (compared to $7.5 \%$ in our series). Similar to our study, the majority of these cancers were identified in the region surrounding the ROI.

The iSR'obot Mona Lisa was introduced by Ho et al. in 2011 to shorten the learning curve of MFB and increase the accuracy (16). The technology has undergone a number of improvements which includes elastic fusion and the first report of this newer version was by Kaufmann et al. (17) A further study by the same group have shown a high CDR of $61 \%$ for MRI suspicious ROIs (9). It is unclear whether robotic-assisted RA-TB is any better than standard MRIUS fusion systems and a large prospective study would be required to answer this. However robotic assistance by the Mona Lisa has the advantage of standardising the procedure with reproducible quality, independent of skill sets. With regards to targeting in particular, it is the only system with robotic control of the depth the biopsy needle fires, ensuring the suspicious lesion is in the middle of the biopsy core. While this study was performed during the initial learning curve of the robotic platform, overall times for the biopsy were actually significantly less (24vs. $32 \mathrm{~min})$ than C-TB biopsies. This however did not include the initial planning time required to contour the prostate and ROIs lesions but was typically performed during patient changeover and did not increase total procedure times.

The other differences are related to the complications following the procedure. With RA-TB, only two perineal punctures are made and the biopsy needle is angled towards different sites in the prostate with RA. This can increase patient comfort and potentially decrease the trauma within the prostate which results in prostate swelling (and acute urinary retention) and haematuria from multiple perforations of the urethra. The results of this study show that both retention rates and prolonged haematuria were significantly lower, supporting this hypothesis.

One of the strengths of this study is that all the MRIs were reported by only one experienced uro-radiologist $(\mathrm{P}$ Vladica). Variability in reporting between observers has been significant even with the PI-RADS version 2 (18), so having consistent MRI PI-RADS version 2 reporting by one radiologist would decrease variability in reporting and make comparison of biopsy techniques more uniform. The same is also true for the single surgeon performing both types of targeted biopsy. Variability in experience in reading MRIs is removed as a potential confounder. All pathology was also reported by one experienced uro-pathologist, again 
decreasing variability seen in reporting.

This study has a number of limitations which need to be considered when assessing the reported outcomes. Firstly, the study has a small number of patients and is limited to two centres, so the outcomes may not be generalisable. This serves as a pilot study to further explore the difference in targeting accuracy between C-TB and RA-TB, which will need confirmation with a larger multicentre randomised trial. A second limitation was that the patients were not randomised but instead had the procedure based on available equipment at their hospital. There was a slight bias towards larger ROIs and higher PSAs in the C-TB group which would have the effect of potentially increasing the CDR in this group, hence true differences between RA-TB and C-TB may be even larger. A third limitation is that this study was performed during the learning curve for operating the RA system and it is quite plausible that there may be a larger difference in targeted biopsy CDR with more operational experience.

Previous studies have not demonstrated a significant difference between C-TB and MF-TB CDRs. This study has shown a significantly higher csPC detection rate and all cancer CDR when MRI ROIs are targeted using RA-TB in comparison to C-TB. This difference may be related to the robotic assistance provided by the Mona Lisa system. RA prostate biopsy also results in lower urinary retention rates and haematuria.

\section{Acknowledgments}

Funding: None.

\section{Footnote}

Conflicts of Interest: All authors have completed the ICMJE uniform disclosure form (available at http://dx.doi. org/10.21037/tau.2020.01.33). The authors have no conflicts of interest to declare.

Ethical Statement: The authors are accountable for all aspects of the work in ensuring that questions related to the accuracy or integrity of any part of the work are appropriately investigated and resolved. This study involves human participants and was approved by The Macquarie University human Ethics Committee approved this study (No. 5201700919).

Open Access Statement: This is an Open Access article distributed in accordance with the Creative Commons Attribution-NonCommercial-NoDerivs 4.0 International License (CC BY-NC-ND 4.0), which permits the noncommercial replication and distribution of the article with the strict proviso that no changes or edits are made and the original work is properly cited (including links to both the formal publication through the relevant DOI and the license). See: https://creativecommons.org/licenses/by-nc-nd/4.0/.

\section{References}

1. Ahmed HU, El-Shater Bosaily A, Brown LC, et al. Diagnostic accuracy of multi-parametric MRI and TRUS biopsy in prostate cancer (PROMIS): a paired validating confirmatory study. Lancet 2017;389:815-22.

2. Kasivisvanathan V, Rannikko AS, Borghi M, et al. MRITargeted or Standard Biopsy for Prostate-Cancer Diagnosis. N Engl J Med 2018;378:1767-77..

3. Wegelin O, van Melick HHE, Hooft L, et al. Comparing Three Different Techniques for Magnetic Resonance Imaging-targeted Prostate Biopsies: A Systematic Review of In-bore versus Magnetic Resonance Imaging-transrectal Ultrasound fusion versus Cognitive Registration. Is There a Preferred Technique? Eur Urol 2017;71:517-31.

4. Wysock JS, Rosenkrantz AB, Huang WC, et al. A prospective, blinded comparison of magnetic resonance (MR) imaging-ultrasound fusion and visual estimation in the performance of MR-targeted prostate biopsy: the PROFUS trial. Eur Urol 2014;66:343-51.

5. Grummet J. How to Biopsy: Transperineal Versus Transrectal, Saturation Versus Targeted, What's the Evidence? Urol Clin North Am 2017;44:525-34.

6. Stefanova V, Buckley R, Flax S, et al. Transperineal Prostate Biopsies Using Local Anesthesia: Experience with 1,287 Patients. Prostate Cancer Detection Rate, Complications and Patient Tolerability. J Urol 2019;201:1121-6.

7. Hoeks CM, Barentsz JO, Hambrock T, et al. Prostate cancer: multiparametric MR imaging for detection, localization, and staging. Radiology 2011;261:46-66.

8. Barrett T, Turkbey B, Choyke PL. PI-RADS version 2: what you need to know. Clin Radiol 2015;70:1165-76.

9. Mischinger J, Kaufmann S, Russo GI, et al. Targeted vs systematic robot-assisted transperineal magnetic resonance imaging-transrectal ultrasonography fusion prostate biopsy. BJU Int 2018;121:791-8.

10. Hansen N, Patruno G, Wadhwa K, et al. Magnetic Resonance and Ultrasound Image Fusion Supported 
Transperineal Prostate Biopsy Using the Ginsburg Protocol: Technique, Learning Points, and Biopsy Results. Eur Urol 2016;70:332-40.

11. Kuru TH, Wadhwa K, Chang RT, et al. Definitions of terms, processes and a minimum dataset for transperineal prostate biopsies: a standardization approach of the Ginsburg Study Group for Enhanced Prostate Diagnostics. BJU Int 2013;112:568-77.

12. Clavien PA, Barkun J, de Oliveira ML, et al. The ClavienDindo classification of surgical complications: five-year experience. Ann Surg 2009;250:187-96.

13. Epstein JI, Egevad L, Amin MB, et al. The 2014 International Society of Urological Pathology (ISUP) Consensus Conference on Gleason Grading of Prostatic Carcinoma: Definition of Grading Patterns and Proposal for a New Grading System. Am J Surg Pathol 2016;40:244-52.

14. Pokorny MR, de Rooij M, Duncan E, et al. Prospective study of diagnostic accuracy comparing prostate cancer

Cite this article as: Patel MI, Muter S, Vladica P, Gillatt D. Robotic-assisted magnetic resonance imaging ultrasound fusion results in higher significant cancer detection compared to cognitive prostate targeting in biopsy naive men. Transl Androl Urol 2020;9(2):601-608. doi: 10.21037/tau.2020.01.33 detection by transrectal ultrasound-guided biopsy versus magnetic resonance (MR) imaging with subsequent MRguided biopsy in men without previous prostate biopsies. Eur Urol 2014;66:22-9.

15. Miah S, Servian P, Patel A, et al. A prospective analysis of robotic targeted MRI-US fusion prostate biopsy using the centroid targeting approach. J Robot Surg 2020;14:69-74.

16. Ho H, Yuen JS, Mohan P, et al. Robotic transperineal prostate biopsy: pilot clinical study. Urology 2011;78:1203-8.

17. Kaufmann S, Mischinger J, Amend B, et al. First report of robot-assisted transperineal fusion versus off-target biopsy in patients undergoing repeat prostate biopsy. World J Urol 2017;35:1023-9.

18. Muller BG, Shih JH, Sankineni S, et al. Prostate Cancer: Interobserver Agreement and Accuracy with the Revised Prostate Imaging Reporting and Data System at Multiparametric MR Imaging. Radiology 2015;277:741-50. 